\title{
Significance of Insluin-Like Growth Factor II mRNA Binding Protein 3 (IMP3) Expression In Selected Thyroid Lesions
}

\author{
Taghreed A. Abd ElAziz, Ranih Z. Amer, Omnia Y. Bassyoni, Hanan A. Ahmed
}

\begin{abstract}
a
Department of pathology,

Benha University, Egypt

Correspondence to: Hanan

A. Ahmed, Department of pathology, Benha University, Egypt

Email:

hanan_abdelaziz20002000

@yahoo.com
\end{abstract}

Available online:14 May 2020:

\section{Abstract:}

Background: Differentiation of benign from malignant follicular thyroid lesions remains difficult and the ability of molecular markers to differentiate between them still unclear. The aim of this study was to evaluate the usefulness of IMP3 expression to distinguish benign from malignant thyroid lesions. Methods: This is a retrospective study upon selected 57 thyroid lesions designated as; 7 cases of Hashimoto thyroiditis (HT), 10 cases of hyperplastic nodules (HN), 15 cases of follicular thyroid adenoma (FTA), 13 cases of conventional papillary thyroid carcinoma (PTC), 6 cases of follicular variant of papillary thyroid carcinoma (FV-PTC), and 6 cases of follicular thyroid carcinoma (FTC). Immunohistochemistry was applied on formalin-fixed, paraffin-embeded tissue blocks using IMP3. clinicopathological data were reviewed from patients' pathological reports and correlated with IMP3 expression. Results: IMP3 positivity was seen in $1 / 7$ cases $(14.3 \%)$ of HT, 2/10 cases (20\%) of HN, 4/15 cases (26.7\%) of FTA, 12/13 cases of PTC, and in all $(100 \%)$ FV-PTC \& FTC cases. IMP3 staining was significantly increased from normal thyroid tissue up to malignant tumors $(\mathrm{P}<0.01)$. IMP3 showed $96 \%$ sensitivity and $78.1 \%$ specificity for malignant tumors. IMP3 expression was positively correlated with grade and tumor size in malignant cases $(\mathrm{P}<0.05)$. No significant correlation was found in IMP3 expression with patient age, sex, capsular invasion, lymphatic/vascular invasion, lymph node metastasis, distant metastasis and TNM stage. Conclusions: IMP3 is a potential diagnostic marker for thyroid cancer and can be a promising marker for distinguishing benign from malignant follicular patterned thyroid lesions.

Keyword: Thyroid lesions, insulin-like growth factor, IMP3. 


\section{Introduction:}

Thyroid nodules, the most common presenting symptom of thyroid cancer, affect $3-4 \%$ of normal population, most thyroid nodules are benign and only $5 \%$ to $15 \%$ is thyroid cancer. ${ }^{1}$ One of greatest challenges in thyroid pathology is differentiation between benign and malignant follicular-patterned neoplasms. When confronted by a follicular patterned tumour, the pathologist relies on capsular and/or vascular invasion to determine biological behaviour of a thyroid tumour.

Multiple studies have demonstrated great inter-observer and intra-observer variability in histopathological diagnosis of follicular pattern thyroid lesions. This variability is because agreement about minimal criteria required for changes in nuclear features of PTC, as well as exact criteria for capsular invasion, not universally accepted. Therefore, the search for objective markers of thyroid malignancy is of great importance. ${ }^{3.4}$

A growing number of some promising immunohisto-chemical markers for the differential diagnosis of thyroid lesions have emerged, including CD56, HBME-1, galectin3 and CK19 but till now none of them are conclusive. $^{5,6}$

Insulin-like growth factor II mRNA-binding protein 3 (IMP3) is a member of the IMP family, consisting of IMP1-3, that plays an important role in RNA traffking, stabilization and translation during embryogenesis. IMP3 promote tumor cell proliferation, migration, invasion, and aggressivenes. ${ }^{7}$ IMP3 has been showed as an important cancer-specific gene associated with several types of cancers including lung cancer, ${ }^{8}$ germ cell cancer, ${ }^{9}$ colon cancer, ${ }^{10}$ pancreatic cancer, ${ }^{11}$ gastric cancer, ${ }^{\mathbf{1 2}}$ liver cancer, ${ }^{\mathbf{1 3}}$ and kidney cancer. ${ }^{14}$ In several organ systems, IMP3 expression has been shown to correlate with a higher grade of tumor, increased risk of metastases, and poorer prognosis. ${ }^{15}$

Depending on the fact that IMP3 is detected in different cancer types at high levels, we conducted this study to evaluate the diagnostic utility of IMP3 in differentiating benign and malignant thyroid lesions, its role in thyroid carcinogenesis and its potential prognostic role.

\section{Material and Methods:}

This study was conducted retrospectively on 63 selected thyroid cases including 6 cases of apparently normal thyroid tissue (as a control group) and 57 cases of different thyroid lesions as a study group, with available formalin-fixed, paraffin-embedded tissue blocks, collected from Archives of Pathology Department and early cancer detection unit of Faculty of Medicine, Benha university during the period from April 2013 to July 2017. 
Hematoxylin and eosin-stained slides on all cases were reviewed by two observers simultaneously to confirm the diagnosis and to classify the lesions into one of the study categories. Thyroid tumors were classified as stated in the WHO classification. ${ }^{\mathbf{1 6}}$ The studied 57 thyroid lesions were classified as 7 cases of HT, 10 cases of HN, 15 cases of FTA, 13 cases of PTC, 6 cases of FV-PTC, and 6 cases of FTC. 6 cases of apparently normal thyroid tissue as a control group were also evaluated. At this review, blocks were selected for immunohistochemistry (IHC).

The remarkable microscopic features such as presence or absence of capsular Invasion (CI), presence or absence of lymphatic/ vascular invasion (LVI), and lymph node status were noted. The patients' demographic and macroscopic data were obtained from their original files, including patient's age, sex, primary lesion site, primary lesion size and distant metastasis status. TNM staging was performed for malignant in accordance to AJCC staging system. ${ }^{17}$

We categorized cases into 3 groups; nonneoplastic group (HT and $\mathrm{HN}$ ), benign follicular neoplasms (FTA) and malignant follicular neoplasm (PTC, FV-PTC, and FTC), and were classified according to age into two groups $<55$ years and $\geq 55$ years, and according to size into three groups $<2 \mathrm{~cm}, 2-4$ $\mathrm{cm}$ and $>4 \mathrm{~cm}$, as shown in (Table 1).

Table (1): Patients' demographic and clinicopathological data in studied groups:

\begin{tabular}{|c|c|c|c|c|}
\hline & $\begin{array}{c}\text { Non-neoplastic lesions } \\
\text { N. }(\%) \\
\end{array}$ & $\begin{array}{c}\text { Benign neoplasm } \\
\text { N. }(\%) \\
\end{array}$ & $\begin{array}{c}\text { Malignant neoplasm } \\
\text { N. }(\%) \\
\end{array}$ & P value \\
\hline \multicolumn{5}{|l|}{ Age } \\
\hline$<55$ years & $16(94.1 \%)$ & $15(100 \%)$ & $21(84 \%)$ & $>0.05$ \\
\hline$\geq 55$ years & $1(5.9 \%)$ & 0 & $4(16 \%)$ & \\
\hline \multicolumn{5}{|l|}{ Sex } \\
\hline Female & $15(88.2 \%)$ & $15(100 \%)$ & $21(84 \%)$ & $>0.05$ \\
\hline Male & $2(11.8 \%)$ & 0 & $4(16 \%)$ & \\
\hline \multicolumn{5}{|l|}{ Site } \\
\hline Right & $6(35.3 \%)$ & $11(73.3 \%)$ & $14(56 \%)$ & \\
\hline Left & $4(23.5 \%)$ & $4(26.7 \%)$ & $5(20 \%)$ & $>0.05$ \\
\hline Bilateral & $7(41.2 \%)$ & 0 & $6(24 \%)$ & \\
\hline \multicolumn{5}{|l|}{ Size } \\
\hline$<2 \mathrm{~cm}$ & $2(11.8 \%)$ & 0 & $4(16 \%)$ & \\
\hline $2-4 \mathrm{~cm}$ & $9(52.9 \%)$ & $11(73.3 \%)$ & $16(64 \%)$ & $>0.05$ \\
\hline$>4 \mathrm{~cm}$ & $6(35.3 \%)$ & $4(26.7 \%)$ & $5(20 \%)$ & \\
\hline Total & $17(100 \%)$ & $15(100 \%)$ & $25(100 \%)$ & \\
\hline
\end{tabular}


Immunohistochemical study: Three micron tissue sections were obtained from formalinfixed, paraffin-embedded tissue blocks on coated slides. After xylene deparaffinization, the sections were rehydrated in descending grades of alcohol then in water. Antigen retrieval was performed by using $10 \mathrm{mmol} / \mathrm{L}$ citrate monohydrate buffer (PH 6.0) and heated for 15 minutes in the microwave. The sections were then incubated in a blocking medium $(3 \% \mathrm{H} 2 \mathrm{O} 2)$ for 15 minutes followed by washing with distilled water. The slides then were immunostained for IMP3 Rabbit polyclonal antibody $(0.1 \mathrm{mg} / \mathrm{ml}$ concentration, Chongqing, 400039, China) at a dilution of 1:100, at room temperature overnight. Immunodetection was executed using a standard labeled streptavidin-biotin system (DakoCytomation, Denmark, A/S). Immune staining was performed based on manufacturer's instructions. Immunoreaction was visualized by adding DAB as a chromagen. Counterstaining of slides was performed with the Mayer hematoxylin.

Normal thyroid tissue present on nearly every slide served as an internal negative control. Fetal liver obtained from aborted fetus (16 weaks), was used as positive control.

Immunostaining evaluation: The slides were evaluated for the presence or absence of IHC staining in lesional and normal thyroid tissue; IMP3 expression was detected as cytoplasmic or membranous brownish coloration. Immunoreactivity was assessed by evaluating extent of stained follicular cells and was considered positive if more than $10 \%$ of follicular epithelial cells showed cytoplasmic/ or membranous staining. The IHC staining was graded as follow; negative (up to $10 \%$ stained cells), focally positive (+: $11-25 \%)$, moderately positive $(++: 26-50 \%)$, or diffusely positive $(+++:>50 \%)$. Expression of IMP3 was then correlated with age, sex, size, CI, LVI, lymph node status, distant metastasis status and TNM stage.

Statistical analysis: Results were analyzed using SPSS (version 16) statistical package for Microsoft windows. The Pearson correlation coefficient was used for statistical analysis, $\mathrm{P}$ value $<0.05$ was considered statistically significant, and highly statistically significant when it was <0.01. Sensitivity, specificity, positive predictive value and negative predictive value were calculated for benign versus malignant lesions. Receiver operating characteristic (ROC) curves were then drawn. The area under the curve (AUC) in ROC analysis was used to compare the ability of a marker to discriminate between benign and malignant lesions.

Results:

Clinico-pathological features in malignant groups: In malignant cases, 5 cases $(38.5 \%)$ of PTC were grade I (GI), 7 cases $(53.8 \%)$ were grade II (GII), and 1 case $(7.7 \%)$ was 
grade III (GIII). 2 cases (33.3\%) of FV-PTC were GI and 4 cases $(66.7 \%)$ were GII. Of FTC cases, 2 cases $(33.3 \%)$ were GI, 2 cases (33.3\%) were GII, and 2 cases $(33.3 \%)$ were GIII. This was not statistically significant $(\mathrm{P}>0.05)$.

When CI was compared between the subtypes of malignant cases, CI was observed in 3 cases $(23.1 \%)$ of PTC,1case $(16.7 \%)$ of FVPTC, and in $100 \%$ of FTC. This was statistically highly significant $(\mathrm{P}<0.01)$. LVI was found in 8 cases (61.5\%) of PTC, 3 cases (50\%) of FV-PTC and 4 cases $(66.7 \%)$ of FTC, but was statistically non significant ( $\mathrm{P}>0.05)$. Lymph node metastasis was found in 8 cases $(61.5 \%)$ of PTC and 3 cases $(50 \%)$ of FV-PTC, but was absent in all cases (100\%) of FTC, and this was statistically significant $(\mathrm{P}<0.05)$. The majority of cases (84\%) were stage I; 10 cases $(76.9 \%)$ of PTC, all cases (100\%) of FV-PTC, and 5 cases $(83.3 \%)$ of FTC were stage I, but this was not statistically significant $(\mathrm{P}>0.05)$

\section{IMP3 staining results: (Table 2) (Figure 1)}

All the control group cases were negative for IMP3 staining •

In non-neoplastic group, 6 cases $(85.7 \%)$ of HT were negative for IMP3 staining and 1 case $(14.3 \%)$ which showed Hurthle cell metaplasia was positive $(++) .8$ cases $(80 \%)$ of $\mathrm{HN}$ were negative and 2 cases $(20 \%)$ were positive (one of 2 cases was formed of oncocytic "Hurthle" cells and showed diffuse cytoplasmic staining for IMP3 (+++). Presence of Hurthle cells in HT \& HN was statistically highly significant $(\mathrm{P}<0.01)$.

In FTA, 11 cases $(73.3 \%)$ were negative, 2 cases $(13.3 \%)$ were positive $(++)$, and 2 cases (13.3\%) which showed high atypical features were diffusely positive $(+++)$, and this was statistically highly significant $(\mathrm{P}<0.01)$.

In malignant cases, 12 cases (92.3\%) of PTC cases were positive with 7 cases $(53.8 \%)$ showed diffuse positive staining (+++). All the 6 cases $(100 \%)$ of FV-PTC and FTC were positive for IMP3.

We found statistically highly significant difference in IMP3 expression between studied cases $(\mathrm{P}<0.01)$ as IMP3 expression was the highest in malignant cases and lowest in non-neoplastic cases and was completely absent in normal thyroid tissue.

IMP3 expression was compared between benign lesions (HT, HN, \&FTA) and malignant lesions (PTC, FV-PTC, \& FTC), and a statistically highly significant difference was found between 2 groups $(\mathrm{P}<0.01)$. We found non statistically significant difference between non-neoplastic lesions and FTA $(\mathrm{P}>0.05)$. When malignant tumors were compared with each others, we found non statistically significant difference $(\mathrm{P}>0.05)$.

Correlation of IMP3 staining with clinicopathological characteristics of studied cases is presented in (Table 2). 
Table (2): IMP3 expression and correlation with clinico/histopathological features in studied cases:

\begin{tabular}{|c|c|c|c|c|c|c|}
\hline & \multirow[t]{2}{*}{ Total } & \multicolumn{4}{|c|}{ Expression of IMP3 [N (\%)] } & \multirow[t]{2}{*}{$P$ value } \\
\hline & & Negative $(0)$ & Focal (+) & Moderate $(++)$ & Diffuse $(+++)$ & \\
\hline \multicolumn{7}{|c|}{ Non-neoplastic lesions } \\
\hline HT & 7 & $6(85.7)$ & 0 & $1(14.3)$ & 0 & $<0.01^{\text {** }}$ \\
\hline $\mathrm{HN}$ & 10 & $8(80)$ & $1(10)$ & 0 & $1(10)$ & - \\
\hline \multicolumn{7}{|c|}{ Benign neoplasms } \\
\hline FTA & 15 & $11(73.3 \%)$ & 0 & $2(13.3 \%)$ & $2(13.3 \%)$ & - \\
\hline \multicolumn{7}{|c|}{ Malignant neoplasms } \\
\hline PTC & 13 & $1(7.7 \%)$ & $2(15.4 \%)$ & $3(23.1 \%)$ & $7(53.8 \%)$ & - \\
\hline FV-PTC & 6 & 0 & $1(16.7 \%)$ & $2(33.3 \%)$ & $(50 \%)$ & - \\
\hline FTC & 6 & 0 & 0 & $2(33.3 \%)$ & $4(66.7 \%)$ & - \\
\hline \multicolumn{7}{|l|}{ Age (years) } \\
\hline$<55$ & 52 & $25(48.1 \%)$ & $4(7.7 \%)$ & $9(17.3 \%)$ & $14(26.9 \%)$ & $>0.05$ \\
\hline$\geq 55$ & 5 & $1(20 \%)$ & 0 & $2(40 \%)$ & $2(40 \%)$ & - \\
\hline \multicolumn{7}{|l|}{ Sex } \\
\hline Female & 51 & $25(49 \%)$ & $2(3.9 \%)$ & $8(15.7 \%)$ & $16(31.4 \%)$ & $>0.05$ \\
\hline Male & 6 & $1(16.7)$ & $2(33.3)$ & $3(50)$ & 0 & - \\
\hline \multicolumn{7}{|l|}{ Grade } \\
\hline Gl & 9 & $1(11.1)$ & $3(33.3)$ & $2(22.2)$ & $3(33.3)$ & $<0.05^{*}$ \\
\hline Gll & 13 & 0 & 0 & $4(30.8)$ & $9(69.2)$ & - \\
\hline GIII & 3 & 0 & 0 & $1(33.3)$ & $2(66.7)$ & - \\
\hline \multicolumn{7}{|l|}{ LVI } \\
\hline Absent & 10 & 0 & $3(30)$ & $1(10)$ & $6(60)$ & $>0.05$ \\
\hline Present & 15 & $1(6.7)$ & 0 & $6(40)$ & $8(53.3)$ & - \\
\hline \multicolumn{7}{|l|}{$\mathrm{Cl}$} \\
\hline Absent & 15 & $1(6.7)$ & $2(13.3)$ & $5(33.3)$ & $7(46.7)$ & $>0.05$ \\
\hline Present & 10 & 0 & $1(10)$ & $2(20)$ & $7(70)$ & - \\
\hline \multicolumn{7}{|l|}{ Tumor size } \\
\hline $\mathrm{T} 1$ & 6 & $1(16.7)$ & $2(33.3)$ & $1(16.7)$ & $2(33.3)$ & $<0.05^{*}$ \\
\hline $\mathrm{T} 2$ & 14 & 0 & $1(7.1)$ & $6(42.9)$ & $7(50)$ & - \\
\hline T3 & 5 & 0 & 0 & 0 & $5(100)$ & - \\
\hline \multicolumn{7}{|c|}{ Nodal metastasis } \\
\hline No & 14 & 0 & $3(21.4)$ & $3(21.4)$ & $8(57.2)$ & $>0.05$ \\
\hline $\mathrm{N} 1$ & 11 & $1(9.1)$ & 0 & $4(36.4)$ & $6(54.5)$ & - \\
\hline \multicolumn{7}{|c|}{ Distant metastasis } \\
\hline MO & 24 & $1(4.2)$ & $3(12.5)$ & $6(25)$ & $14(58.3)$ & $>0.05$ \\
\hline M1 & 1 & 0 & 0 & $1(100)$ & 0 & - \\
\hline \multicolumn{7}{|c|}{ Tumor stage (TNM) } \\
\hline Stage I & 21 & $1(4.8)$ & $3(14.3)$ & $5(23.8)$ & $12(57.1)$ & $>0.05$ \\
\hline Stage II & 3 & 0 & 0 & $1(33.3)$ & $2(66.7)$ & - \\
\hline Stage III & 0 & 0 & 0 & 0 & 0 & - \\
\hline Stage IV & 1 & 0 & 0 & $1(100)$ & 0 & - \\
\hline
\end{tabular}




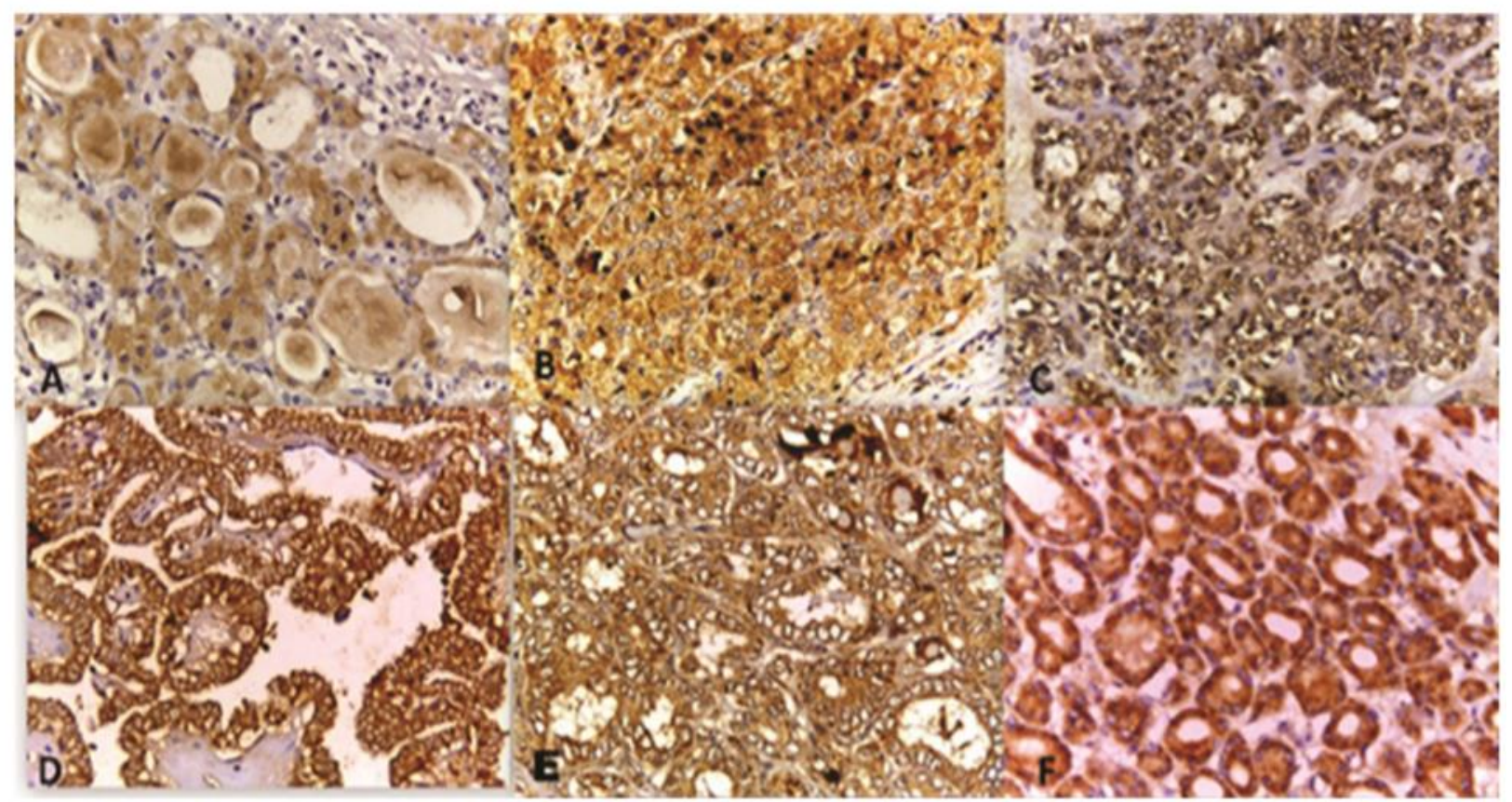

Figure 1: Representative micrographs of immunohistochemical staining for IMP3 in different thyroid lesions. Positive staining for IMP3 in Hashimoto thyroiditis with Hurthle cells ++ (A), diffuse positive cytoplasmic staining in Hurthle cell hyperplastic nodule +++ (B), positive cytoplasmic staining in Follicular thyroid adenoma $++(\mathrm{C})$, diffuse positive cytoplasmic and membranous staining in conventional papillary thyroid carcinoma $+++(D)$, diffuse positive staining in follicular variant-papillary thyroid carcinoma +++ (E), and Follicular thyroid carcinoma +++ (F), X400

We found statistically significant difference in IMP3 expression in relation to tumor grade and size $(\mathrm{P}<0.05)$. There was no statistically significant difference in IMP3 expression in relation to age, sex, CI, LVI, lymph node status, distant metastasis and stage $(\mathrm{P}>0.05)$.

\section{Receiver operating characteristic curve} and area under the curve analysis for

\section{IMP3 expression in benign versus malignant thyroid lesions:}

The ability of IMP3 to distinguish benign from malignant thyroid lesions was thereafter determined by means of ROC analysis. The values of the area under the ROC curve (AUC) are shown in (Table 3) and the actual curves are shown in (Figure 2)

For malignant tumors, the sensitivity of IMP3 was $96 \%$, and specificity was $78.1 \%$. Comparison of FTA with FTC and FTA with FV-PTC revealed $100 \%$ sensitivity and $73.3 \%$ specificity when IMP3 is used to differentiate between these lesions. Comparison of $\mathrm{HN}$ with FV-PTC revealed $100 \%$ sensitivity and $80 \%$ specificity. Finally, comparison of $\mathrm{HN}$ with PTC revealed $92.3 \%$ sensitivity and $80 \%$ specificity. (Table 3) 
Table (3): Statistical analysis of IMP3 as a marker for malignancy for follicular patterned thyroid lesions:

\begin{tabular}{lccccc}
\hline & Sensitivity & Specificity & PPV & NPV & AUC \\
\hline $\begin{array}{l}\text { Benign lesions }{ }^{*} \text { vs. } \\
\text { malignant tumors }\end{array}$ & $96 \%$ & $78.1 \%$ & $77.4 \%$ & $96.2 \%$ & 0.886 \\
FTA vs. FTC & $100 \%$ & $73.3 \%$ & $60 \%$ & $100 \%$ & 0.889 \\
FTA vs. FV-PTC & $100 \%$ & $73.3 \%$ & $60 \%$ & $100 \%$ & 0.856 \\
HN vs. FV-PTC & $100 \%$ & $80 \%$ & $75 \%$ & $100 \%$ & 0.917 \\
HN vs. PTC & $92.3 \%$ & $80 \%$ & $85.7 \%$ & $88.9 \%$ & 0.881
\end{tabular}

N.B: Benign lesions (includes non-neoplastic lesions and follicular thyroid adenoma)

PPV Positive predictive value, NPV Negative predictive value, AUC Area under the curve, FTA Follicular thyroid adenoma, FTC Follicular thyroid carcinoma, FV-PTC follicular variant of papillary thyroid carcinoma, HN Hyperplastic nodules, PTC Papillary thyroid carcinoma.
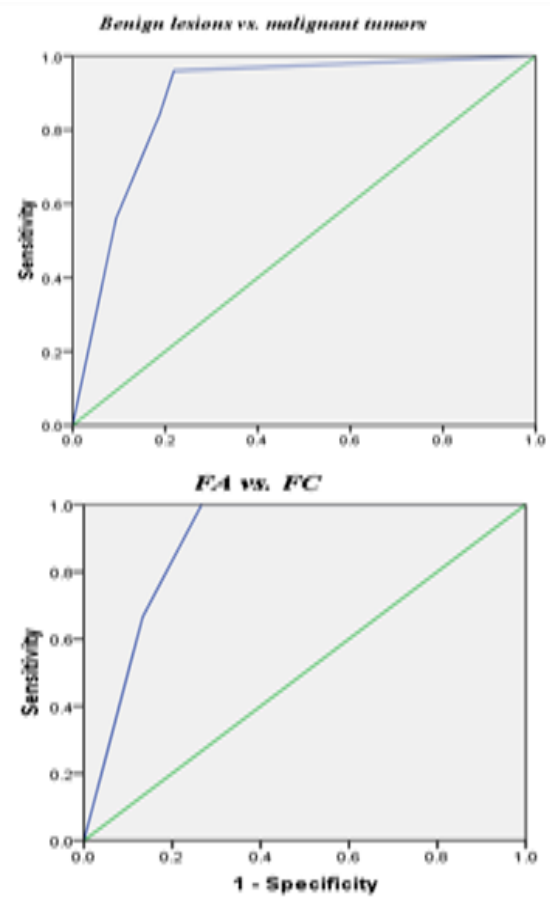
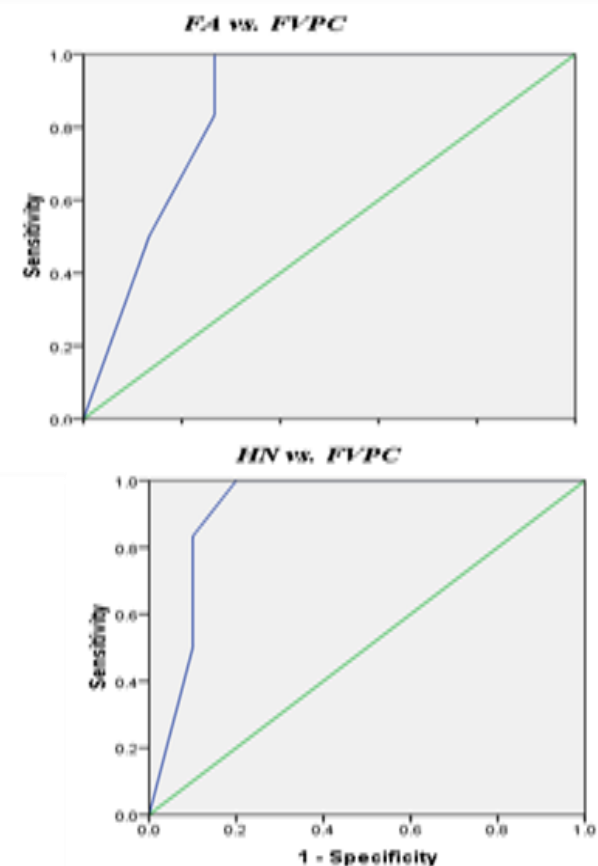

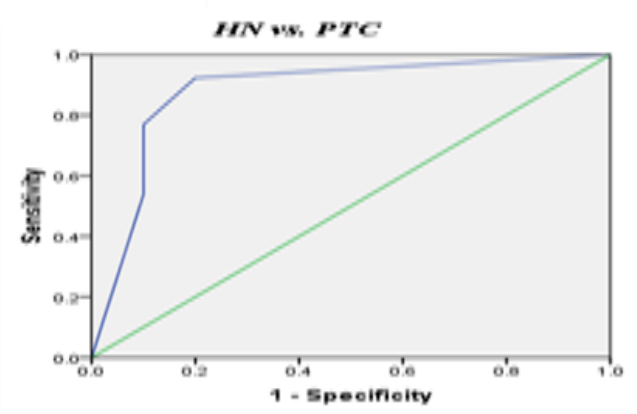

Figure (2): Receiver operating characteristic analysis for IMP3. The area under the curve represents an optimal statistic for comparing the sensitivity and specivicity of IMP3 for differentiating between benign and malignant thyroid lesions. N.B: FA Follicular adenoma, FVPC Follicular variant of papillary carcinoma, FC Follicular carcinoma, HN Hyperplastic nodules, PTC Papillary thyroid carcinoma. 
Discussion:

IMP3 promotes tumor cell growth, proliferation, survival and tumor cell invasiveness in vitro, through the control of the translation or turnover of various candidate target genes, including IGF2, CD44, HMGA2, and MMP9. ${ }^{\mathbf{1 8 - 2 0}}$ In our study we aimed to evaluate the expression of IMP3 in different thyroid lesions and to evaluate its usefulness as a diagnostic marker in differentiating benign and malignant thyroid lesions and its possible prognostic role in malignant thyroid lesions.

In present study IMP3 expression significantly increased gradually from apparently normal thyroid tissue (100\% negative expression) to non-neoplastic lesions ( $17.6 \%$ positivity), to benign neoplasms ( $26.7 \%$ positivity) to be the highest in malignant neoplasms $(94 \%$ positivity) ( $\mathrm{P}$ value $<0.01$ ). Our finding were compatible with the results of other series in literature evaluating the expression of IMP3 in thyroid lesions. ${ }^{\mathbf{2 1 - 2 4}}$ This indicates that IMP3 may be implicated in thyroid carcinogensis by initiating malignant transformation and proliferation of transformed cells. Also, we demonsrated that IMP3 is a useful marker for differenting benign from malignant thyroid lesions, with $96 \%$ sensitivity and $78.1 \%$ specificity for malignant lesions.

In the studied 7 cases of hashimoto thyroiditis, 6 cases $(85.7 \%)$ were negative for
IMP3 expression and only 1 case (14.3\%) was positive (++). This is consistent with the work of Jin et al. $(2015)^{25}$, who observed positive expression for IMP3 in 50\% of HT cases.

In hyperplastic nodules, 8 cases $(80 \%)$ were negative for IMP3 expression and only 2 cases $(20 \%)$ were positive $(+\&+++)$. This is in agreement with the results of El-Shorbagy et al. $(2015)^{23}$ and Kulaçoğlu and Erkılınç $(\mathbf{2 0 1 5})^{24}$, who demonstrated positive staining for IMP3 in $25 \%$ and $50 \%$ of their studied $\mathrm{HN}$, respectively.

In contrast, Slosar et al. (2009) $)^{21}$ found complete negative IMP3 staining in HT and $\mathrm{HN}$ cases. This can be explained by technical variation and different antibodies clones used.

From these results, it was demonstrated that pre-existing benign thyroid disease is one of the risk factors for development of thyroid malignancy. In line with this statement, Resende de Paiva $(2017)^{26}$ studied association between HT and thyroid cancer in 64,628 patients, and demonstrated a high association between HT and thyroid cancer, mostly PTC. Also it can be demonstrated that thyroid is a site for hyperplasia-neoplasia sequence as it is now evident that clonal proliferations occur in some $\mathrm{HN}$ and can express various markers of malignant follicular derived thyroid tumours such as 
PPAR, RET19 and activating RAS mutations. $^{27,28}$

In studied 15 cases of FTA, 11 cases (73.3\%) were negative and 4 cases $(26.7 \%)$ were positive for IMP3. Close to our results Jin et al. $(2010)^{22}$ and El-Shorbagy et al. $(2015)^{23}$, also observed positive staining in $11.1 \%$ and $25 \%$ of FTA cases, respectively, while Slosar et al., (2009) $)^{22}$, found complete negativity for IMP3 staining in FTAs, which disagree with our findings.

Positive expression for IMP3 in FTA confirms the biological relationship between FTA and FTC as FTA shares FTC in the same genetic alteration (e.g. chromosomal abnormalities, activating RAS mutations, and PAX8/PPARG rearrangements). ${ }^{29}$

In studied malignant group, we found a positive expression for IMP3 in 12/13 cases (92.3\%) of PTC, and in all cases $(100 \%)$ of FV-PTC and FTC, with the highest and strongest expression was seen in FTC cases. Our results were parallel to results of other studies evaluating IMP3 expression in differentiated thyroid carcinomas. ${ }^{23,45,30}$

In study of Slosar et al. (2009) $)^{21}$, they found a rare and weak staining for IMP3 in PTC group, only 4 out of 37 cases (11\%) were positive, which was incompatible with our results, and on the other hand, they found a strong expression for IMP3 in follicular variant of papillary carcinoma and follicular carcinoma (38\% \& 69\%, respectively), which is compatible with our findings.

In this work, we studied the diagnostic performance of IMP3 in thyroid lesions by comparing its expression between different studied groups and performing its sensitivity and specificity. We found a statistically highly significant difference in IMP3 expression between benign lesions (HT, HN and FTA) and malignant lesions $(\mathrm{P}<0.01)$, and between benign neoplasms (FTA) and malignant neoplasms $(\mathrm{P}<0.01)$. on the other hand there was non significant difference in IMP3 expression between non-neoplastic lesions and FTA $(P>0.05)$, and between malignant tumors when compared with each other $(P>0.05)$ in terms of positive staining.

In this study, IMP3 showed; 100\% sensitivity and $73.3 \%$ specificity in differentiating FTC from FTA, 100\% sensitivity and specificity (73.3 and 80\%) in distinguishing FV-PTC from FTA and $\mathrm{HN}$, respectively, and in distinguishing HN from PTC, IMP3 showed $92.3 \%$ sensitivity and $80 \%$ specificity. Collectively IMP3 has $96 \%$ sensitivity and $78.1 \%$ specificity for malignant lesions.

Parallel to our finding, Slosar et al. (2009) ${ }^{21}$, found that IMP3 had 100\% specificity for FTC and FV-PTC as compared with FTA and 69\% sensitivity for FTC also compared with FTA. Similarly, Jin et al. (2010) $)^{22}$, on performing IMP-3 qRT-PCR analysis, they 
documented a $91.4 \%$ specificity and $86.7 \%$ sensitivity for diagnosis of well-differentiated thyroid carcinomas.

In contrast, in study of Kulaçoğlu and Erkılınç (2015) ${ }^{24}$, although they observed

$82.1 \%$ positivity for IMP3 in malignant cases, they found non significant difference between benign lesions and malignant tumors. Also they found a higher frequency of positivity in benign lesions (66.7\%) both in HN (50\%) and FTA (92.9\%) with a statistically significant difference between them $(\mathrm{P}<0.05)$, which is incompatible with our results.

Our results suggest that IMP3 could be a magic marker for differentiation of benign and malignant thyroid lesions especially follicular patterned lesions which can represent a diagnostic challenge, and it can be a complementary marker between a panel used to improve the sensitivity and specificity of an individual marker in differentiating benign and malignant follicular lesions of thyroid.

In current work, we found a significantly increased IMP3 expression in malignant tumors with higher grade and larger size $(\mathrm{P}<0.05)$. This was in agreement with results of Gao et al. (2014) $)^{13}$, who reported a significant correlation between IMP3 expression and intrahepatic cholangiocarcinoma with larger tumor size and higher grade, so it can be reported that
IMP3 expression not associated only with initiation of malignancy but also with its progression and poor prognosis. In contrast, Kulaçoğlu and Erkılınç $(2015)^{24}$ and Yorukoglu et al. $(2015)^{30}$, reported non significant correlation between IMP3 expression and tumor size and grade in thyroid malignancy.

Correlation of IMP3 expression with other clinico-pathological parameters including age, sex, LVI, CI, lymph node status and TNM stage, we found no clear cut correlation between these parameters and IMP3 positivity $(\mathrm{P}>0.05)$, this was in accordance with studies by Kulaçoğlu and Erkılınç (2015) ${ }^{24}$ and Yorukoglu et al. $(2015)^{30}$.

In study performed by Asioli et al. (2010) ${ }^{31}$, IMP3 expression was compared with the Clinic-pathologic parameters in poorly differentiated carcinomas, and they found that IMP3 expreassion was related to increased death risk, lymph node metastasis, and distant metastasis, this can be explained by their study was only on poorly differentiated carcinomas and their use of different methods for IMP3 detection.

In study of Slosar et al. (2009) ${ }^{21}$, they stated that IMP3 expression increased in differentiated thyroid carcinoma with larger tumor size, higher frequency of LVI, and lymph node metastases, but their results did not reach a stastistical significant difference. 
Also they found higher expression for IMP3 in PTC with nodal metastasis, but owing to small number of positive cases in this group they couldn't draw any definite conclusions.

Given contradictory findings in different studies, further studies with larger number of cases and long-term follow-up may be useful to establish prognostic significance of IMP3 in thyroid carcinoma.

Conclusion: IMP3 may be involved in thyroid carcinogenesis, the initiation, proliferation and progression of tumor cells. IMP3 is a potential diagnostic marker for thyroid cancer, and can be a promising marker for differentiating follicular patterened thyroid lesions.IMP3 expression increases with increase size and grade of malignant thyroid tumors, which indicates a possible prognostic role for IMP3 in thyroid cancer.

\section{References:}

1- Haugen BR, Alexander EK, Bible KC, Doherty GM, Mandel SJ, Nikiforov YE, et al. 2015 American Thyroid Association Management Guidelines for Adult Patients with Thyroid Nodules and Differentiated Thyroid Cancer: The American Thyroid Association Guidelines Task Force on Thyroid Nodules and Differentiated Thyroid Cancer. Thyroid 2016; 26:1-133.

2- Siegel RL, Miller KD, and Jemal A. Cancer statistics, 2018. CA: a cancer journal for clinicians 2018; 68:7-30

3- Baloch ZW and LiVolsi VA. Follicular-patterned afflictions of the thyroid gland: reappraisal of the most discussed entity in endocrine pathology. Endocrine pathology 2014; 25:12-20

4- Asa SL. The evolution of differentiated thyroid cancer. Pathology 2017; 49:229-37

5- Paunovic I, Isic T, Havelka M, Tatic S, Cvejic D, and Savin S. Combined immunohistochemistry for thyroid peroxidase, galectin-3, CK19 and HBME-1 in differential diagnosis of thyroid tumors. Apmis 2012; 120:368-79.

6- Alshenawy HA. Utility of immunohistochemical markers in differential diagnosis of follicular cellderived thyroid lesions. J Microsc Ultras 2014; 2:127-36.

7- Bell JL, Wachter K, Muhleck B, Pazaitis N, Köhn M, Lederer $\mathbf{M}$, et al. Insulin-like growth factor 2 mRNAbinding proteins (IGF2BPs): posttranscriptional drivers of cancer progression? Cell Mol Life Sci 2013; 70:2657-75.

8- Yan J, Wei Q, Jian W, Qiu B, Wen J, Liu J, et al. IMP3 predicts invasion and prognosis in human lung adenocarcinoma. Lung 2016; 194:137-46.

9- Goodman S, Zhang L, Cheng L, and Jiang Z. Differential expression of IMP3 between male and female mature teratomas immunohistochemical evidence of malignant nature. Histopathology 2014; 65:483-9.

10- Wei Q, Zhou H, Zhong L, Shi L, Liu J, Yang Q, et al. IMP3 expression in biopsy specimens as a diagnostic biomarker for colorectal cancer. Human pathology 2017; 64:137-44.

11- Lok T, Chen L, Lin F, and Wang HL. Immunohistochemical distinction between intrahepatic cholangiocarcinoma and pancreatic ductal adenocarcinoma. Hum Pathol 2014; 45:394400.

12- Damasceno EAM, Carneiro FP, Magalhães AV, de Vasconcelos Carneiro M, Takano GHS, de Sousa Vianna LM, et al. IMP3 expression in gastric cancer: Association with clinicopathological 
features and HER2 status. J Cancer Res Clin Oncol 2014; 140:2163-68.

13- Gao Y, Yang M, Jiang Z, Woda BA, Mercurio AM, Qin J, et al. IMP3 expression is associated with poor outcome and epigenetic deregulation in intrahepatic cholangiocarcinoma. Human pathology 2014; 45:1184-91.

14- Pei X, Li M, Zhan J, Yu Y, Wei X, Guan L, et al. Enhanced IMP3 Expression Activates NF-кB Pathway and Promotes Renal Cell Carcinoma Progression. PLoS ONE 2015; 10:e0124338

15- Burdelski C, Jakani-Karimi N, Jacobsen F, Möller-Koop C, Minner S, Simon R, et al. IMP3 overexpression occurs in various important cancer types and is linked to aggressive tumor features: A tissue microarray study on 8,877 human cancers and normal tissues. Oncology reports 2018; 39:3-12.

16- Lam AKY. Pathology of Endocrine Tumors Update: World Health Organization New Classification 2017-Other Thyroid Tumors. AJSP: Reviews \& Reports 2017; 22:209-16.

17- Amin MB, EdgeS, Greene F, Byrd DR, Brookland RK, Washington MK, et al. AJCC Cancer Staging Manual, 8th edn. American Joint Committee on Cancer 2017.

18- Li W, Liu D, Chang W, Lu X, Wang YL, Wang H, et al. Role of IGF2BP3 in trophoblast cell invasion and migration. Cell Death Dis 2014; 5:e1025.

19- Jønson L, Christiansen J, Hansen TV, Vikeså J, Yamamoto Y, and Nielsen FC. IMP3 RNP safe houses prevent miRNA-directed HMGA2 mRNA decay in cancer and development. Cell Rep 2014; 7:539-51.

20- Bellezza G, Prosperi E, Del Sordo R, Colella R, Rulli A, and Sidoni A. IMP3 is strongly expressed in malignant phyllodes tumors of the breast: an immunohistochemical study. International journal of surgical pathology 2016; 24:37-42.
21- Slosar M, Vohra P, Prasad M, Fischer A, Quinlan $\mathrm{R}$, and Khan A. Insulin-like growth factor mRNA binding protein 3 (IMP3) is differentially expressed in benign and malignant follicular patterned thyroid tumors. Endocrine pathology 2009; 20:149-57.

22- Jin L, Seys AR, Zhang S, Erickson-Johnson MR, Roth CW, Evers BR, et al. Diagnostic utility of IMP3 expression in thyroid neoplasms: a quantitative RT-PCR study. Diagnostic Molecular Pathology 2010; 19:63-9.

23- El-Shorbagy SH, El-Azeem MAA, and El-Azeem MAA. Differentiation of benign from malignant follicular-cell-derived thyroid lesions: an immunohistochemical study. Egyptian Journal of Pathology 2015; 35:119-32.

24- Kulac,og lu S and Erkılınc, G. Imp3 expression in benign and malignant thyroid tumors and hyperplastic nodules. Balkan Med J 2015; 32:30 7.

25- Jin L, Lloyd RV, Henry MR, Erickson LA, Sebo TJ, Rumilla KM, et al. The diagnostic utility of combination of HMGA2 and IMP3 qRT-PCR testing in thyroid neoplasms. Applied immunohistochemistry \& molecular morphology $2015 ; 23: 36-43$.

26- Resende de Paiva C, Grønhøj C, Feldt-Rasmussen $\mathrm{U}$, and von Buchwald C. Association between Hashimoto's thyroiditis and thyroid cancer in 64,628 patients. Frontiers in Oncology 2017; 7:110.

27- Paschke R. Molecular pathogenesis of nodular goiter. Langenbecks Arch Surg. 2011; 396:112736.

28- Khadim MT, Ashraf HM, and Zehra T. Clinicomorphological spectrum of thyroid lesions with follicular pattern on microscopy. Pak Armed Forces Med J 2017; 67:69-73.

29- Tallini G and Giordano TJ. Thyroid gland. In: Goldblum JR: Rosai and Ackerman's Surgical 
Pathology. 11th edition, Elsevier Health Sciences 2018; Ch.8:278-354.

30- Yorukoglu A, Yalcin N, Avci A, Cakalagaoglu F, Yaylali G, Akin F, et al. Significance of IMP3, nucleophosmin, and $\mathrm{Ki}-67$ expression in papillary thyroid carcinoma. International journal of surgical pathology 2015; 23:5-12.
31- Asioli S, Erickson LA, Righi A, Jin L, Volante M, Jenkins S, et al. Poorly differentiated carcinoma of the thyroid: validation of the Turin proposal and analysis of IMP3 expression. Modern Pathology 2010; 23:1269-78.

To cite this article: Taghreed A. Abd ElAziz, Ranih Z. Amer, Omnia Y. Bassyoni, Hanan A. Ahmed.Significance of insulin-like growth factor II mRNA binding protein 3 expression in selected thyroid lesions. BMFJ. 2020;37(1):305-318. 